УДК 621.396 .96

\title{
ОЦЕНКА ЭФФЕКТИВНОСТИ ДИСКРЕТНЫХ АЛГОРИТМОВ АДАПТАЦИИ ВЕСОВЫХ КОЭФФИЦИЕНТОВ ПРИ ПРОСТРАНСТВЕННО-ВРЕМЕННОЙ ОБРАБОТКЕ РАДИОЛОКАЦИОННЫХ СИГНАЛОВ
}

\author{
Д. М. ПИЗА ${ }^{1}$, Д. С. СЕМЕНОВ ${ }^{2}$, С. В. МОРЩАВКА ${ }^{1}$ \\ ${ }^{1}$ Запорожский национальный технический университет, \\ Украина, Запорожье, 69063, ул. Жуковского, 64 \\ ${ }^{2}$ Казенное предприятие «Научно-производственный комплекс «Искра», \\ Украина, Запорожье, 69071, ул. Магистральная, 84
}

\begin{abstract}
Аннотация. Проведен сравнительный анализ эффективности работы адаптивных пространственных фильтров при различных методах формирования классифицированной обучающей выборки в условиях одновременного воздействия активных шумовых и пассивных помех. На основании предложенной методики проведены аналитические расчеты изменений межканальных фазовых сдвигов при дискретных алгоритмах адаптации весовых коэффициентов пространственного фильтра в различных режимах работы РЛС 36Д6. Показано, что при сканировании антенной системы на интервалах пауз в адаптации весовых коэффициентов возникают межканальные фазовые рассогласования, величина которых зависит от угловой скорости вращения антенной системы и от периода повторения импульсов РЛС. Проведена оценка коэффициента подавления, реализуемого в конце паузы в адаптации весовых коэффициентов с учетом межканального фазового рассогласования. Разработаны рекомендации по выбору метода формирования классифицированной обучающей выборки в условиях воздействия комбинированных помех с учетом потерь, возникающих при дискретной адаптации весовых коэффициентов пространственного фильтра.
\end{abstract}

Ключевые слова: адаптивный пространственный фильтр; комбинированная помеха; радиолокационная станция; межканальное рассогласование; коэффициент подавления помехи

\section{ВВЕДЕНИЕ}

В реальных условиях работы на радиолокационную станцию (РЛС) могут воздействовать как активные шумовые, так и пассивные помехи [1-3]. Активная помеха создается расположенными в дальней зоне антенны точечными по дальности и угловым координатам источниками шумовых излучений. Пассивная помеха порождается отражениями собственного зондирующего сигнала РЛС от местных предметов, гидрометеоров, дипольных помех.
При комбинированном воздействии активных шумовых помех (АШП) и переотражений от пассивных отражателей пространственно-распределенный характер пассивной помехи разрушает пространственную корреляцию активной составляющей комбинированной помехи. На участках дальности в угловых направлениях, где пассивная составляющая комбинированной помехи является преобладающей, компенсация активной помехи может оказаться проблематичной. Кроме того, наличие пассивной помехи приводит к существенному затягиванию переходных процессов при 
адаптации пространственных или поляризационных фильтров [4].

Таким образом, для эффективной компенсации активных помех необходимо сформировать классифицированную обучающую выборку (КОВ), порожденную только активной составляющей комбинированной помехи. Однако, формирование КОВ путем выбора интервала дальности, на котором пассивная помеха отсутствует, с последующим ее запоминанием, и использованием на остальных интервалах, приводит к наличию пауз в адаптивной настройке системы защиты от активных помех, что ограничивает эффективность системы помехозащиты. Поэтому оценка потерь, возникающих при дискретной адаптации систем помехозащиты, является достаточно актуальной.

Цель работы состоит в анализе эффективности систем пространственно-временной обработки сигналов при дискретной адаптации весовых коэффициентов.

\section{ПОСТАНОВКА ЗАДАЧИ}

Оптимальная фильтрация полезных сигналов в условиях воздействия комбинированных помех при использовании адаптивных антенных решеток может быть обеспечена совместной параллельной (не разделяющейся) пространственно-временной обработкой с одновременной компенсацией активных шумовых и пассивных помех. Однако ее реализация при большом количестве пространственных каналов даже на современной элементной базе крайне затруднительна [5]. Поэтому при проектировании современных радиолокационных станций обнаружения, сопровождения и целеуказания для автоматизированных систем управления используют раздельную, последовательную двухэтапную процедуру обработки радиолокационных сигналов. При этом в качестве элемента адаптации пространственных фильтров используют известные автокомпенсаторы с корреляционной обратной связью или с прямым вычислением весовых коэффициентов [6]. Однако в условиях воздействия комбинированных помех при независимой адаптации пространственного и временного фильтра потери системы помехозащиты в выходном отношении сигнал/(помеха + шум) достигают 10-20 дБ [7].

На основании анализа множества работ, посвященных адаптации пространственно-временных фильтров, в [7] сформирован вывод, что несмотря на постановку задачи реализации взаимозависимой адаптации пространственного и временного фильтров конструктивных решений получено не было.

Задачей, решаемой в статье, является оценка эффективности системы помехозащиты при пространственно-временной взаимозависимой обработке сигналов [7] с учетом дискретной адаптации весовых коэффициентов пространственного фильтра при формировании КОВ.

\section{ОБЗОР ЛИТЕРАТУРЫ}

Для формирования КОВ, порожденной только АШП, могут быть использованы априорные или апостериорные методы. Во многих известных технических решениях для формирования КОВ предложено использовать фиксированный временной интервал, расположенный в конце дальности действия РЛС. Предложенные технические решения основаны на априорном предположении, что интенсивность пассивной помехи по мере увеличения дальности уменьшается. При этом наличие фиксированного интервала упрощает реализацию метода.

Однако при пространственно-распределенных кучево-дождевых облачных системах типа $\mathrm{Cb}$ интенсивность отраженных сигналов на предельной дальности действия РЛС в нижних угломестных каналах зоны обзора может существенно превышать собственные шумы приемного устройства. Это не позволяет гарантированно обеспечить эффективную ком- 
пенсацию активной составляющей комбинированной помехи.

В $[8,9]$ предложены дискретные методы формирования классифицированной обучающей выборки, использующие естественную нестационарность пассивной помехи во времени (по дальности). Это характерно для сигналов, отраженных как от гидрометеоров, так и от облаков дипольных отражателей, имеющих локальный характер. Предложенные методы основаны на использовании апостериорной информации относительно распределения пассивной помехи по дальности.

С учетом того, что при одновременном воздействии активной шумовой помехи и пассивной помехи последняя декоррелирует сигналы, порожденные точечным источником активной помехи, представляется возможным оценить распределение модуля межканального коэффициента корреляции (ММКК) на интервалах дальности. При этом по известным значениям ММКК выбирается временной интервал для адаптации весовых коэффициентов автокомпенсатора помех (АКП) с максимальным значением межканальной корреляции, который соответствует наименьшему уровню (или отсутствию) пассивной помехи.

ММКК $|\dot{\rho}|$ может быть рассчитан по методу «скользящего окна» на интервалах, состоящих из $m$ дискретов дальности:

$$
|\dot{\rho}|=\frac{\overline{\left|\dot{U}_{0} \dot{U}_{k}^{*}\right|}}{\sqrt{\overline{\left|\dot{U}_{0}\right|^{2}} \mid \overline{\left.\dot{U}_{k}^{*}\right|^{2}}}}
$$

где $\dot{U}_{0}-$ комплексное значение помехи на входе основного канала автокомпенсатора, $\dot{U}_{k}^{*}$ - комплексно-сопряженное значение помехи на входе компенсационного канала.

Количество дискретов $m$, подлежащих усреднению в (1), определяется временем адаптации весовых коэффициентов пространственного фильтра. Если в выражении для вычисления весового коэффициента АКП
$\dot{k}=\overline{\dot{U}_{0} \dot{U}_{k}^{*}}\left(\overline{\dot{U}_{k} \dot{U}_{k}^{*}}\right)^{-1}$, а также в выражении для ММКК (1) интервалы усреднения принять одинаковыми, то вычисление весового коэффициента и ММКК выполняются параллельно и синхронно. Таким образом, обеспечивается возможность работы РЛС в режиме реального времени. Например, при длительности полезного сигнала 1 мкс длительность переходного процесса при формировании весового коэффициента, а соответственно, и формирование ММКК, может составлять порядка 10 мкс.

В [9] предложен следующий метод формирования КОВ. Вычисленные по выражению (1) значения ММКК подаются на пороговое устройство. Если ММКК принимает значение, которое превышает установленный порог, то на выходе порогового устройства формируется сигнал, разрешающий формирование весовых коэффициентов АКП. Реализуется такой алгоритм путем использования запоминающего устройства, включенного между формирователем весовых коэффициентов и регулируемым усилителем (умножителем) автокомпенсатора. В данном случае при превышении ММКК установленного порога запоминающее устройство переходит в линейный режим передачи весовых коэффициентов, поступающих на вход регулируемого усилителя.

Если вычисленное значение ММКК становится меньше установленного порога, то запоминающее устройство фиксирует сформированное ранее значение весовых коэффициентов для компенсации АШП на интервале действия пассивной помехи. Если в текущем периоде повторения значение ММКК снова превышает пороговый уровень, то запоминающее устройство переходит в линейный режим передачи весовых коэффициентов, что обеспечивает очередную их оптимизацию в том же периоде повторения и т.д.

ММКК функционально связан с коэффициентом подавления помех, поэтому уровень порога можно выбрать по величине минималь- 


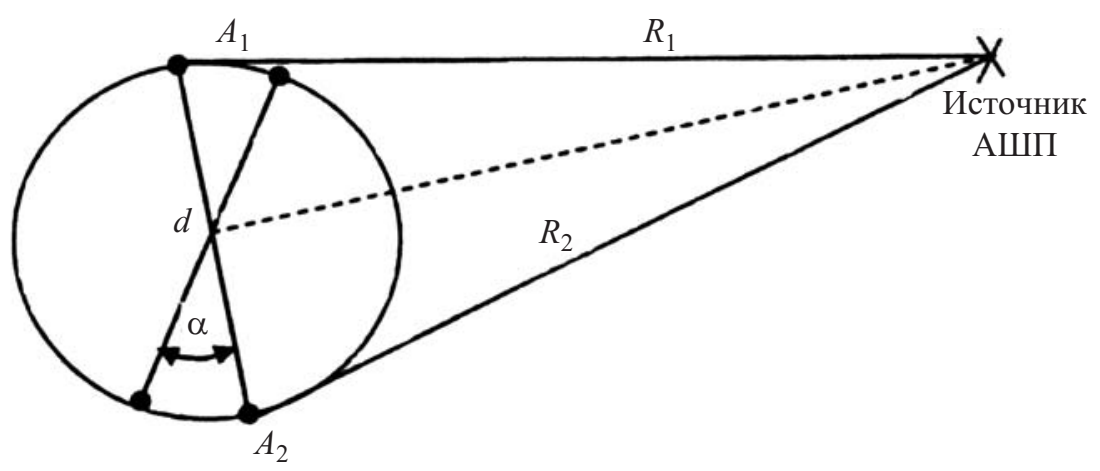

Рис. 1. Схема приема АШП на разнесенную антенную систему.

но допустимого значения коэффициента подавления, например, равного 3 дБ. Это обусловлено возрастающими флуктуациями весового коэффициента АКП при уменьшении ММКК, что приводит к модуляции пассивной помехи, действующей в компенсационном канале. Последнее ограничивает обнаружение полезных сигналов на фоне пассивных помех во второй ступени пространственно-временной обработки.

В [8] предложен метод, обеспечивающий детальный анализ распределения ММКК по дальности, который заключается в следующем. Вычисленные по выражению (1) значения ММКК запоминаются в цифровой многоотводной линии задержки. В конце периода повторения зондирующих импульсов все вычисленные значения ММКК поступают в устройство выбора максимума, где определяется интервал с наибольшим значением ММКК. В дальнейшем формирование весовых коэффициентов автокомпенсатора выполняется именно на этом интервале.

Представлялось целесообразным провести сравнительную оценку эффективности адаптивных пространственных фильтров при использовании предложенных методов формирования КОВ с учетом потерь, возникающих при дискретной адаптации весовых коэффициентов.

\section{МЕТОДИКА ОЦЕНКИ}

При защите РЛС от активных помех, действующих по боковым лепесткам диаграммы направленности антенны, использование пространственных отличий между полезным сигналом и помехой реализуется путем разнесенного приема. При этом между основной и компенсационной антеннами существует расстояние, называемое базой. Следствием использования дискретной адаптации весовых коэффициентов пространственного фильтра является наличие пауз в самонастройке автокомпенсаторов. В процессе сканирования антенной системы РЛС проекция базы на направление источника активной помехи изменяется. Это приводит к изменению межканальных фазовых сдвигов во время пауз. При этом сформированные весовые коэффициенты становятся не оптимальными. В конечном счете, это приводит к ухудшению эффективности пространственной фильтрации. Поэтому представляется необходимым оценить потери в компенсации АШП при использовании рассмотренных методов формирования КОВ и сформировать рекомендации к проектированию систем помехозащиты.

Схема приема активной шумовой помехи антенной системой, состоящей из двух антенн: основной антенны $A_{1}$ и компенсационной антенны $A_{2}$, фазовые центры которых разнесены на расстояние $d$, представлена на рис. 1. Расстояния от источника АШП до фазовых центров антенн обозначены как $R_{1}$ и $R_{2}$. 
Таблица 1

\begin{tabular}{||l|c|c|c|c|c|c||}
\hline \hline Период повторения $T_{\text {п }}, \mathrm{mc}$ & \multicolumn{2}{|c|}{0,66} & \multicolumn{2}{|c||}{1,33} & \multicolumn{2}{|c||}{2,66} \\
\hline Период обзора $T_{\text {об, }}$ с & 5 & 10 & 5 & 10 & 5 & 10 \\
\hline Смещение антенной системы $\Delta$, мин & 2,83 & 1,42 & 5,70 & 2,87 & 11,3 & 5,74 \\
\hline Изменение проекции базы $\Delta d$, мм & 1,24 & 1 & 2,89 & 1,25 & 64,2 & 2,98 \\
\hline Изменение межканального фазового сдвига $\varphi_{\text {мк }, \text { град }}$ & 4,46 & 3,60 & 10,4 & 4,50 & 23,1 & 10,4 \\
\hline
\end{tabular}

Рассмотрим как изменяется проекция базы $d$ на направление источника помехи при вращении антенной системы. Будем считать, что в случае, представленном на рис. 1 , при $R_{1}=R_{2}$, помеховые сигналы поступают на антенны $A_{1}$ и $A_{2}$ синфазно и межканальный фазовый сдвиг $\varphi_{\text {мк }}$ отсутствует. При этом проекция базы $d$ на нормаль к антенной системе равна нулю.

Будем считать, что при повороте антенной системы в процессе сканирования на угол $\alpha=5^{\circ}$ активная помеха действует в направлении первых боковых лепестков диаграммы направленности основной антенны. При этом нарушается условие $R_{1}=R_{2}$, что приводит к изменению межканальных фазовых сдвигов.

В процессе исследований разработана методика оценки межканальных фазовых сдвигов, которые возникают при дискретной адаптации весовых коэффициентов пространственного фильтра и влияют на качество подавления помех. Методика включает три этапа:

- на первом этапе определяется угловое смещение антенной системы $\Delta$ на интервале времени, соответствующем длительности паузы при дискретной адаптации весовых коэффициентов пространственного фильтра;

- на втором этапе вычисляется соответствующее изменение проекции базы $\Delta d$ на направление источника активной помехи на том же временном интервале;

- на третьем этапе, с учетом рабочей длины волны РЛС, выполняется пересчет величины проекции базы $\Delta d$ в межканальный фазовый сдвиг $\varphi_{\text {мк }}$, по которому определяется эффективность пространственной фильтрации [10].

В качестве исходных данных для оценки потерь, обусловленных изменением межканальных фазовых сдвигов на интервале пауз в формировании весовых коэффициентов, использованы параметры РЛС отечественного производства 36Д6. При этом длительность периода обзора $T_{\text {об }}=5$ или $10 \mathrm{c}$, длительность периода повторения зондирующих импульсов $T_{\text {п }}$ $=0,66 ; 1,33 ; 2,66$ мс, длительность сигнала $\tau_{\text {и }}=$ 1,$5 ; 3,0 ; 6,0$ мкс, соответственно, база $d=2 \mathrm{м}$.

\section{РЕЗУЛЬТАТЫ}

Выполним расчет углового смещения $\Delta$ антенной системы на интервале, равном периоду повторения $T_{\text {п }}=0,66$ мс при периоде обзора $T_{\text {об }}=5 \mathrm{c}$ по формуле:

$$
\Delta=\frac{2 \pi \rho}{T_{\text {об }}} T_{\text {п }},
$$

где сомножитель $2 \pi \rho / T_{\text {об }}$ [рад/с] представляет собой скорость вращения антенной системы. При выводе (2) учтено, что длительность паузы при дискретных методах адаптации [8, 9] намного больше интервала формирования КОВ, поэтому длительность паузы приравнена к периоду повторения $T_{\text {п }}$.

При заданных исходных данных угловое смещение антенной системы составляет: 


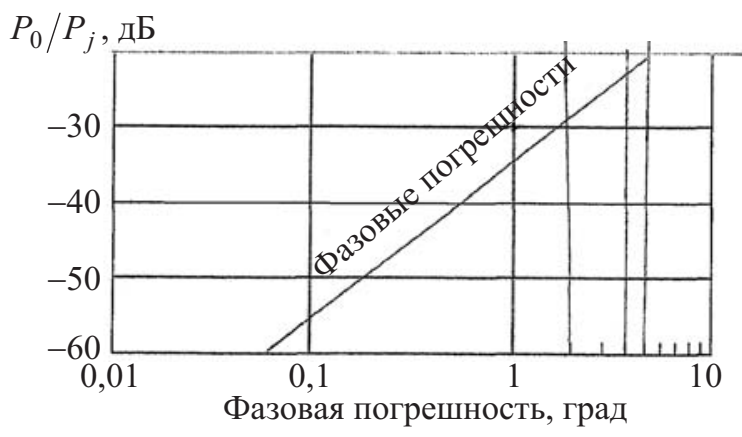

Рис. 2. Зависимость нормированного нескомпенсированного остатка $P_{j}$ от межканального рассогласования.

$$
\Delta=\frac{6,28 \rho}{5} \times 0,66 \times 10^{-3}=0,829 \times 10^{-3} \rho=2,83^{\prime}
$$

В процессе расчета аналогично определены значения углового смещения антенной системы $\Delta$ при различных значениях периода обзора $T_{\text {об }}$ и периода повторения импульсов $T_{\text {п. }}$ Результаты расчета $\Delta$ при различных режимах работы РЛС приведены в табл. 1.

Далее определялась величина изменения проекции базы $\Delta d$ на направление источника активной помехи в соответствии с формулой:

$$
\Delta d=d \sin \alpha .
$$

Если активная помеха действует в направлении первых боковых лепестков диаграммы направленности основной антенны и угол $\alpha=$ $5^{\circ}$, тогда в соответствии с (3), $\Delta d=17,4$ см. Далее полагая, что начало паузы в адаптации весовых коэффициентов совпадает с направлением $\alpha=5^{\circ}$, определим изменение проекции базы на интервале, равном периоду повторения зондирующих импульсов РЛС. При этом изменение проекции $\Delta d$ на интервале длительности паузы в адаптации весовых коэффициентов составит:

$$
\Delta d=d \sin \left(5^{\circ}+\alpha\right)-\Delta d,
$$

где первое слагаемое определяет проекцию базы в конце периода повторения, а второе - в начале.
Например, если период повторения равен 0,66 мс и $T_{\text {об }}=5 \mathrm{c}$, то $\Delta=2,83^{\prime}$. При этом $\Delta d=$ $2 \sin \left(5^{\circ}+2,83^{\prime}\right)-0,174=1,24$ мм, или с учетом длины волны 0,1 м, изменение межканального фазового сдвига к концу паузы в адаптации весовых коэффициентов составит $4,46^{\circ}$. Результаты расчета изменения межканального фазового сдвига $\varphi_{\text {мк }}$ при различных режимах работы РЛС приведены в табл. 1.

На рис 2 изображена зависимость нескомпенсированного остатка $P_{0}$, нормированного по мощности АШП $P_{j}$, от межканального фазового рассогласования [9].

Сопоставление полученных в конце паузы значений межканального фазового сдвига $\varphi_{\text {мк }}$ со значениями, приведенными на рис. 2, дает основание считать, что только при $T_{\text {п }}=$ 0,66 мс $\left(T_{\text {об }}=5\right.$ и $\left.10 \mathrm{c}\right)$, а также при $T_{\text {п }}=1,33$ мс $\left(T_{\text {об }}=10 \mathrm{c}\right)$ величина коэффициента подавления АШП составляет не меньше 20 дБ. С учетом других факторов, ограничивающих эффективность системы помехозащиты от АШП, в этих режимах работы РЛС целесообразно использовать метод формирования КОВ, предложенный в [8]. Это обусловлено тем, что при этом адаптация весовых коэффициентов пространственного фильтра выполняется на единственном временном интервале в течение периода повторения $T_{\text {п }}$, где модуль нормированного межканального коэффициента корреляции имеет максимальное значение. 
В других режимах работы РЛС, т.е. при больших значениях пауз в адаптации весовых коэффициентов, межканальноые фазовые сдвиги не позволяют реализовать достаточно высокие значения коэффициента подавления АШП при использовании метода [8]. Однако, при больших периодах повторения $T_{\text {п }}$ за счет нескольких подстроек весовых коэффициентов на интервалах дальности, свободных от пассивных помех, возможно уменьшить длительность пауз и при этом увеличить коэффициент подавления АШП, что предложено в [9].

Полученные результаты не ограничиваются случаем использования одного источника АШП и одноканальным автокомпенсатором. Известные алгоритмы параллельно-последовательной обработки (в русскоязычной литературе) и алгоритм Пауела (в англоязычной), позволяют спроектировать многоканальную систему помехозащиты с использованием одноканальных автокомпенсаторов. При этом также решается задача ортогонализации помеховых сигналов в компенсационных каналах приема, что повышает эффективность системы помехозащиты при воздействии многих источников АШП.

\section{выводы}

Проведен сравнительный анализ эффективности адаптивных пространственных фильтров при различных методах формирования классифицированной обучающей выборки в условиях одновременного воздействия активных и пассивных помех.

Выполнены аналитические расчеты изменений межканальных фазовых сдвигов при дискретных алгоритмах адаптации весовых коэффициентов пространственного фильтра в различных режимах работы РЛС 36Д6. Показано, что при сканировании антенной системы, на интервалах пауз в адаптации весовых коэффициентов возникают межканальные фазовые рассогласования, величина которых зависит от угловой скорости вращения антенной системы и периода повторения импульсов РЛС.

Разработаны рекомендации по выбору метода формирования классифицированной обучающей выборки в условиях воздействия комбинированных помех с учетом потерь, возникающих при дискретной адаптации весовых коэффициентов пространственного фильтра.

\section{БИБЛИОГРАФИЧЕСКИЙ СПИСОК}

1. Falk, K. "A method and radar system for repetition jammer and clutter suppression," US Patent WO2012067557A1. 27 Oct. 2015.

2. Пиза, Д. М.; Звягинцев, Е. А.; Мороз, Г. В. “Метод компенсации активной составляющей комбинированной помехи в когерентно-импульсной РЛС," Известия вузов. Радиоэлектроника, Т. 59, № 6, С. 23-29, 2016. DOI: $10.20535 / \mathrm{S} 0021347016060030$.

3. Уидроу, Б.; и др. “Адаптивные антенные системы,” ТИИЭР, Т. 55, № 12, С. 78-95, 1967.

4. Абрамович, Ю.И.; Качур, В.Г. “Быстродействие поочередной настройки раздельных систем защиты от комбинированных помех," Радиотехника $и$ электроника, № 1, С. 52-58, 1969.

5. Рябуха, В.П.; Рачков, Д.С.; Семеняка, А.В.; Катюшин, Е.А. “Оценка интервала фиксации пространственного весового вектора при последовательной пространственно-временной обработке сигналов на фоне комбинированных помех," Известия вузов. Радиоэлектроника, Т. 55, № 10, C. 13-25, 2012. DOI: 10.20535/S0021347012100020.

6. Уидроу, Б.; и др. “Адаптивные компенсаторы помех. Принципы построения и применения," ТИИЭР, Т. 63, № 12, С. 69-99, 1975.

7. Григорьев, В.В. Комбинированная обработка сигналов в системах радиосвязи. М.: Эко-Трендз, 2002. $262 \mathrm{c}$.

8. Пиза, Д.М.; Лаврентьев, В.Н.; Семенов, Д.С. "Метод формирования классифицированной обучающей выборки для автокомпенсатора помех при время-пространственной фильтрации сигналов," Радиоэлектроника, информатика, управление, № 3, С. 18-22, 2016. DOI: $10.15588 / 1607-3274-2016-3-2$.

9. Пиза, Д. М.; Мороз, Г. В. “Методы формирования классифицированной обучающей выборки для адаптации весового коэффициента автокомпенсатора помех," Известия вузов. Радиоэлектроника, Т. 61, № 1, C. 47-54, 2018. DOI: $10.20535 / \mathrm{S} 0021347018010041$.

10. Монзинго, Р.А.; Миллер, Т. У. Адаптивные антенные решетки: введение в теорию. Пер. с англ. под ред. В.А. Лексаченко. М.: Радио и связь, 1986. 445 с. 


\section{N F O RMATION ON THE ART ICLE}

\section{EFFICIENCY ESTIMATION OF DISCRETE ALGORITHMS FOR ADAPTATION OF WEIGHT COEFFICIENTS IN SPACE-TIME PROCESSING OF RADAR SIGNALS}

Dmytro M. Piza, ORCID: 0000-0001-9717-512X, dpiza@zntu.edu.ua, Zaporizhzhya National Technical University, Ukraine

Dmytro S. Semenov, State Enterprise "Scientific and Production Complex “Iskra”, Ukraine

Sergii V. Morshchavka, Zaporizhzhya National Technical University, Ukraine

A comparative analysis of the efficiency of adaptive spatial filters with different methods of classified training sample formation under conditions of simultaneous exposure to active noise and passive interference is carried out. Based on the proposed methodology, analytical calculations of the changes in interchannel phase shifts for discrete algorithms for the adaptation of the weight coefficients of the spatial filter in various modes of operation of the 36D6 radar are carried out. It is shown that interchannel phase desynchronization/error arise at pause intervals in the adaptation of weight coefficients during antenna system scanning. The magnitude of these desynchronizations depends on the angular velocity of the antenna system rotation and on the repetition period of the radar pulses. An estimation of the suppression coefficient realized at the end of the pause in the adaptation of the weight coefficients taking into account the interchannel phase desynchronization is carried out. The recommendations on the choice of the formation method of the classified training sample under the conditions of the combined interferences are considered taking into account the losses that arise in case of discrete adaptation of the weight coefficients of the spatial filter.

Keywords: adaptive spatial filter; combined interference; radar; interchannel desynchronization; interference suppression coefficient

\section{REFERENCES}

1. FALK, K. "A method and radar system for repetition jammer and clutter suppression," US Patent WO2012067557A1. 27 Oct. 2015.

2. PIZA, D.M.; ZVIAHINTSEV, Ye.A.; MOROZ, G.V. "Method of compensating the active component of combined interference in coherent pulse radar," Radioelectron. Commun. Syst., v.59, n.6, p.251, 2016. DOI: 10.3103/S07352727160600 $\underline{30 .}$.

3. WIDROW, B.; MANTEY, P.E.; GRIFFITHS, L.J.; GOODE, B.B. "Adaptive antenna systems," Proc. IEEE, v.55, n.12, p.2143, 1967. DOI: 10.1109/PROC.1967.6092.

4. ABRAMOVICH, Yu.I.; KACHUR, V.G. "Speed of response of sequential tuning of separate combined interference compensation systems," Radiotechnika i Elektronika, n.1, p.52, 1969.

5. RIABUKHA, V.P.; RACHKOV, D.S.; SEMENIAKA, A.V.; KATIUSHYN, Ye.A. "Estimation of spatial weight vector fixation interval for sequential space-time signal processing against the background of combined interferences," Radioelectr- on. Commun. Syst., v.55, n.10, p.443, 2012. DOI: $\underline{10.3103 / \mathrm{S} 0}$ 735272712100020 .

6. WIDROW, B.; GLOVER, J.R.; MCCOOL, J.M.; KAUNITZ, J.; WILLIAMS, C.S.; HEARN, R.H.; ZEIDLER, J.R.; DONG, Jr. Eugene; GOODLIN, R.C. "Adaptive noise cancelling: Principles and applications," Proc. IEEE, v.63, n.12, p.1692, 1975. DOI: 10.1109/PROC.1975.10036.

7. GRIGORYEV, V.V. Combined Signal Processing in Radiosystems [in Russian]. Moscow: Eko-Trends, 2002.

8. PIZA, D.M.; LAVRENTIEV, V.N.; SEMENOV, D.S "Method of forming of the classified training sample for automatic canceller of the interferences when using time-space filtering of signals," Radioelectronics, Computer Science, Control, n.3, p.18, 2016. DOI: 10.15588/1607-3274-2016-3-2.

9. PIZA, D.M.; MOROZ, G.V. "Methods of forming classified training sample for adaptation of weight coefficient of automatic interference compensator," Radioelectron. Commun. Syst., v.61, n.1, p.32, 2018. DOI: 10.3103/S0735272718010041.

10. MONZINGO, R.A.; MILLER, T.W. Introduction to Adaptive Arrays. Wiley-Interscience, 1980. 